\title{
High-order gauge-invariant perturbations of a spherical spacetime
}

\author{
David Brizuela, José M. Martín-García, and Guillermo A. Mena Marugán \\ Instituto de Estructura de la Materia, CSIC, Serrano 121-123, 28006 Madrid, Spain
}

(Received 12 March 2007; published 10 July 2007)

\begin{abstract}
We complete the formulation of a general framework for the analysis of high-order nonspherical perturbations of a four-dimensional spherical spacetime by including a gauge-invariant description of the perturbations. We present a general algorithm to construct these invariants and provide explicit formulas for the case of second-order metric perturbations. We show that the well-known problem of lack of invariance for the first-order perturbations with $l=0,1$ propagates to increasing values of $l$ for perturbations of higher order, owing to mode coupling. We also discuss in which circumstances it is possible to construct the invariants.
\end{abstract}

DOI: 10.1103/PhysRevD.76.024004

PACS numbers: 04.25.Nx, 04.20.Cv, 95.30.Sf

\section{INTRODUCTION}

The highly nonlinear character of the Einstein equations of general relativity requires the use of numerical and perturbative techniques to study problems of astrophysical interest. Processes like the merge of binary systems (black holes and/or neutron stars) are promising candidates for gravitational wave detection in the next decade, and need careful and detailed simulation to accurately predict the waveforms that will be observed.

The use of perturbation theory can be important both as a tool for simulating the evolution of a system in a state close to a known solution, or as a tool to extract the information of the amount and form of the gravitational radiation emitted. First-order perturbation theory is normally used in both cases, but it is possible to improve the accuracy of the results by using perturbation theory to higher orders.

We have recently introduced a general framework to study generic high-order nonspherical perturbations of an arbitrary spherical spacetime [1]. It can be considered as a generalization of the formalism of Gerlach and Sengupta (GS) for first-order perturbations around spherical symmetry [2], in the sense that we use the same concepts and techniques: the background spacetime is decomposed as the product of a 2-dimensional Lorentzian manifold and the 2-sphere; covariant notations are used on both submanifolds; and all perturbations are covariantly decomposed as series in tensor harmonics. However, the GS formalism also uses a gauge-invariant description, whereas our presentation in Ref. [1] employed a fixed gauge (the ReggeWheeler gauge [3]). The present article fills in this gap and completes the construction of the high-order generalization of the GS formalism by showing how one can construct gauge-invariant high-order perturbations. This allows one to treat the perturbations in any gauge while dealing with quantities and expressions that present the same form for all physically equivalent perturbations.

The issue of perturbative gauge invariance is closely related, though not equal, to the coordinate independence of general relativity and has been dealt with in different ways already in first-order perturbation theory. The pioneering works by Sachs [4], Moncrief [5], and Stewart and Walker [6] established most of the basic ideas of the subject at first order, but offered different points of view about gauge invariance, what sometimes obscures the interpretation of the results and complicates any attempt of generalizing the analysis to higher orders. Bruni and collaborators have shown [7] that the geometrical approach of Sachs [4], already rather constraining at first order, becomes even more restrictive at higher orders and hence only highly symmetric scenarios can be described in this approach. Here we will show that the alternative approach by Moncrief [5] allows a greater and more convenient flexibility, so that we will adhere to it in order to construct metric gauge invariants at both first and second order around a spherical spacetime.

This article, which can thus be considered a continuation of Ref. [1] (referred to as Paper I in the following), is organized as follows. Section II reviews the concept of gauge transformations and Sec. III discusses the different notions of gauge invariance that have been employed in the literature, introducing a general method for the construction of gauge invariants. Section IV describes the notation adopted for spherically symmetric spacetimes and presents a brief summary on the structure of tensor harmonics (see Paper I for a more detailed description). Section V constructs gauge-invariant nonspherical perturbations around a spherical spacetime, using the method introduced in Sec. III, and is the central section of the paper. Finally, Sec. VI contains our conclusions. Appendix A is devoted to the study of mode coupling and discusses in which cases the high-order gauge invariants can be consistently constructed. A particular case, already studied in the literature [8], is described in Appendix B.

\section{GAUGE FREEDOM IN PERTURBATION THEORY}

Perturbation theory in general relativity considers a family of spacetimes $\left(M_{\varepsilon}, g_{\varepsilon}\right)$ in which associated families of tensor fields $\Omega_{\varepsilon}$ are defined. The $\varepsilon=0$ members of 
those families are referred to as the background members and will be denoted without a subscript, e.g. the background metric is $g \equiv g_{0}$. All manifolds $M_{\varepsilon}$ are assumed to be diffeomorphic. The main issue in perturbation theory is comparing tensor fields for a given nonzero value of $\varepsilon$ with their background counterparts. There exists diffeomorphism invariance on each of the manifolds $M_{\varepsilon}$ but, in addition, there is no preferred point-to-point identification mapping between any two such manifolds, so that the comparison of two tensor fields with different values of $\varepsilon$ is not an invariantly defined concept. This is the origin of the so-called gauge freedom in perturbation theory [6].

Let us call a gauge $\phi_{\varepsilon}$ a family of point-to-point identification diffeomorphisms from the background manifold to $M_{\varepsilon}$ :

$$
\phi_{\varepsilon}: M \rightarrow M_{\varepsilon} .
$$

Given a gauge $\phi_{\varepsilon}$ we can now pull back a generic tensor $\Omega_{\varepsilon}$ on $M_{\varepsilon}$ to a tensor $\phi_{\varepsilon}^{*} \Omega_{\varepsilon}$ on $M$. This latter tensor can be compared with the background member $\Omega$ (at each point $x \in M$ ), resulting in a $\phi$-dependent concept of what a perturbation means. Assuming smooth dependence of all structures in $\varepsilon$, we can define the perturbative expansion

$$
\phi_{\varepsilon}^{*} \Omega_{\varepsilon} \equiv \Omega+\sum_{n=1}^{\infty} \frac{\varepsilon^{n}}{n !} \Delta_{\phi}^{n}[\Omega],
$$

where all terms of the equation are defined at the same point of the background manifold $M$. In particular the perturbations $\Delta_{\phi}^{n}[\Omega]$ are tensor fields on $M$. The notation $\Delta_{\phi}$ stresses the fact that, in general, it is not possible to define a perturbation without explicitly indicating which gauge $\phi$ is used. For instance, the statement that a perturbation vanishes is generically meaningless unless one specifies the gauge in which this occurs. Note also that the infinite series in Eq. (2) arises from the simultaneous dependence on $\varepsilon$ of both $\Omega_{\varepsilon}$ and $\phi_{\varepsilon}$.

One then has to face the question of how the perturbations $\Delta_{\phi}^{n}[\Omega]$ vary under a change of gauge from $\phi_{\varepsilon}$ to, let us say, $\psi_{\varepsilon}$ while keeping unaltered the family of tensors $\Omega_{\varepsilon}$. Such a gauge transformation will be described by a family $\chi_{\varepsilon}$ of diffeomorphisms on the background manifold

$$
\chi_{\varepsilon} \equiv \phi_{\varepsilon}^{-1} \circ \psi_{\varepsilon}: M \rightarrow M,
$$

which clearly satisfy

$$
\psi_{\varepsilon}^{*} \Omega_{\varepsilon}=\chi_{\varepsilon}^{*} \phi_{\varepsilon}^{*} \Omega_{\varepsilon} .
$$

We emphasize that $\chi_{\epsilon}$ is not a gauge, but a gauge transformation.

The theory of expansions of families of general diffeomorphisms on a given manifold has been developed in Ref. [7]. The most important result is that any oneparameter family of diffeomorphisms, in particular, the gauge transformation $\chi_{\varepsilon}$, is equivalent to an infinite set of flows (a flow is a one-parameter group of diffeomor- phisms) $\left\{\Phi_{\varepsilon}^{(1)}, \ldots, \Phi_{\varepsilon^{m} / m !}^{(m)}, \ldots\right\}$, so that $\chi_{\varepsilon}$ can be expressed in the following way

$$
\chi_{\varepsilon}=\ldots \circ{ }^{\{m\}} \Phi_{\varepsilon^{m} / m !} \circ \ldots \circ{ }^{\{2\}} \Phi_{\varepsilon^{2} / 2} \circ{ }^{\{1\}} \Phi_{\varepsilon} .
$$

From the definition of the Lie derivative, it is straightforward to see that the pullback of any flow $\Phi_{\varepsilon}$ acting on a generic tensor $T$ can be expanded as

$$
\Phi_{\varepsilon}^{*} T=\sum_{n=0}^{\infty} \frac{\varepsilon^{n}}{n !} \mathcal{L}_{\xi}^{n} T,
$$

where the vector $\xi$ is the generator of the flow. Making use of this formula and the decomposition (5), we can expand the right-hand side of Eq. (4) in a power series, so that two gauge choices are related by

$$
\begin{aligned}
\psi_{\varepsilon}^{*} \Omega_{\varepsilon}= & \sum_{k_{1}=0}^{\infty} \sum_{k_{2}=0}^{\infty} \ldots \sum_{k_{m}=0}^{\infty} \ldots \frac{\varepsilon^{k_{1}+2 k_{2}+\ldots+m k_{m}+\ldots}}{2^{k_{2}} \ldots(m !)^{k_{m}} \ldots k_{1} ! k_{2} ! \ldots k_{m} ! \ldots} \\
& \times \mathcal{L}^{k_{1}{ }_{\{1\}} \xi} \mathcal{L}^{k_{2}{ }_{\{2\}} \xi} \ldots \mathcal{L}^{k_{m}{ }_{\{m\}}} \ldots \phi_{\varepsilon}^{*} \Omega_{\varepsilon} .
\end{aligned}
$$

The vector fields $\left\{{ }^{\{1\}} \xi,{ }^{\{2\}} \xi, \ldots,{ }^{\{m\}} \xi, \ldots\right\}$ are the corresponding generators of the flows $\left\{{ }^{\{1\}} \Phi,{ }^{\{2\}} \Phi, \ldots\right.$, $\left.{ }^{\{m\}} \Phi, \ldots\right\}$. Substituting expansion (2) in the above equation, we get [9]

$$
\begin{aligned}
\Delta_{\psi}^{n}[\Omega]-\Delta_{\phi}^{n}[\Omega]= & \sum_{m=1}^{n} \frac{n !}{(n-m) !} \sum_{\left(K_{m}\right)} \frac{1}{2^{k_{2}} \ldots(m !)^{k_{m}} k_{1} ! \ldots k_{m} !} \\
& \times \mathcal{L}^{k_{1}{ }_{{ }_{11}} \xi \ldots \mathcal{L}^{k_{m}}{ }_{\{m\}} \Delta_{\phi}{ }_{\phi}^{n-m}[\Omega]}
\end{aligned}
$$

where we have defined

$$
\left(K_{m}\right)=\left\{\left(k_{1}, \ldots, k_{m}\right) \in \mathbb{N}^{m} ; \quad \sum_{i=1}^{m} i k_{i}=m\right\} .
$$

Up to third order we obtain

$$
\begin{gathered}
\Delta_{\psi}[\Omega]-\Delta_{\phi}[\Omega]=\mathcal{L}_{\{1\} \xi} \Omega, \\
\Delta_{\psi}^{2}[\Omega]-\Delta_{\phi}^{2}[\Omega]=\left(\mathcal{L}_{\{2\} \xi}+\mathcal{L}_{\{1\} \xi}^{2}\right) \Omega+2 \mathcal{L}_{\{1\} \xi} \Delta_{\phi}[\Omega],
\end{gathered}
$$

$$
\begin{aligned}
\Delta_{\psi}^{3}[\Omega]-\Delta_{\phi}^{3}[\Omega]= & \left(\mathcal{L}_{\{3\} \xi}+\mathcal{L}^{3}{ }_{\{1\}}+3 \mathcal{L}_{\{1\} \xi} \mathcal{L}_{\{2\}}\right) \Omega \\
& +3\left(\mathcal{L}_{\{2\}}+\mathcal{L}_{\{1\} \xi}^{2}\right) \Delta_{\phi}[\Omega] \\
& +3 \mathcal{L}_{\{1\}} \Delta_{\phi}^{2}[\Omega] .
\end{aligned}
$$

These formulas describe the effect of general gauge transformations on any high-order perturbation of a generic background tensor $\Omega$. They contain all the information needed to analyze the issue of gauge transformations in perturbation theory.

In practical applications we can keep terms only up to a finite order $\varepsilon^{n}$, which projects the full group $G$ of gauge transformations into a truncated group ${ }^{\{n\}} \mathcal{G}$ of $n$ th-order 
gauge transformations. Each of these transformations is described by a collection of $n$ vector fields $\left.\{11\} \xi, \ldots,{ }^{\{n\}} \xi\right\}$, and we will say that it is pure $m$ th order if all those vectors are zero except for ${ }^{\{m\}} \xi$. The important point is that, in general, composition of pure $m$ th-order transformations is not pure $m$ th order, unless $m=n$. For example, the composition of two generic second-order transformations described by $\left\{{ }^{\{1\}} \bar{\xi},{ }^{\{2\}} \bar{\xi}\right\}$ and $\left\{{ }^{\{1\}} \hat{\xi},{ }^{\{2\}} \hat{\xi}\right\}$ is described by the pair

$$
\left\{{ }^{\{1\}} \bar{\xi}+{ }^{\{1\}} \hat{\xi},{ }^{\{2\}} \bar{\xi}+{ }^{\{2\}} \hat{\xi}+\left[{ }^{\{1\}} \bar{\xi},{ }^{\{1\}} \hat{\xi}\right]\right\},
$$

and hence the subset of pure first-order transformations $\left\{{ }^{\{1\}} \boldsymbol{\xi}, 0\right\}$ is not a subgroup of ${ }^{\{2\}} \mathcal{G}$. In fact, the group ${ }^{\{1\}} \mathcal{G}$ is not equivalent to this subset, but only to a truncated form of it, and therefore it is important to distinguish between firstorder transformations $\{\{1\} \xi\}$ and pure first-order transformations $\left\{{ }^{\{1\}} \xi, 0, \ldots 0\right\}$. In general, the set ${ }^{\{n\}} \mathcal{G}$ of all $n$ thorder gauge transformations is a group, but the subset of all pure $m$ th-order transformations is not. The only exception is the reduced case $m=n$ of transformations of the form $\{0, \ldots, 0,\{n\} \xi\}$, in which only a single linear term in Eq. (8) survives (this includes first-order perturbation theory as the case $m=n=1$ ). There are more general subgroups of a given ${ }^{\{n\}} \mathcal{G}$, like the subgroup of transformations of the form $\left\{0, \ldots, 0,{ }^{\{m\}} \xi,{ }^{\{m+1\}} \xi, \ldots,{ }^{\{n\}} \xi\right\}$, but they have less interest for our discussion and will not be considered in this work.

\section{THE NOTION OF GAUGE INVARIANCE}

Once we have defined the concept of gauge transformation in Eq. (3), we discuss now the associated notion of gauge invariance of a family of tensors $\Omega_{\varepsilon}$ under a group of gauge transformations. We will then find the inherited gauge invariance of the perturbations $\Delta_{\phi}^{n}[\Omega]$ under the respective truncated version of that group.

The most natural definition of gauge invariance was given by Sachs [4]: A tensor family $\Omega_{\varepsilon}$ is identification gauge invariant (IGI) if the pullback of its members to the background manifold is independent of the gauge, though the result still depends on $\varepsilon$. That is, $\phi_{\varepsilon}^{*} \Omega_{\varepsilon}=\psi_{\varepsilon}^{*} \Omega_{\varepsilon}$ for all gauges $\phi_{\varepsilon}, \psi_{\varepsilon}$. This can also be interpreted as the invariance under the full group $G$ of gauge transformations. Perturbatively, a tensor family $\Omega_{\varepsilon}$ is IGI up to order $n$ if and only if $\Delta_{\phi}^{m}[\Omega]=\Delta_{\psi}^{m}[\Omega]$ for all $m \leq n$ and all gauges $\phi, \psi[7]$. Again, this is equivalent to the requirement of invariance under the truncated group ${ }^{\{n\}} \mathcal{G}$ defined above. This definition turns out to be too restrictive because, as it is well known $[4,6]$, only perturbations of vanishing tensors, constant scalars, or constant linear combinations of products of delta tensors can be IGI in first-order perturbation theory, since these are the only tensors with zero Lie derivative along every vector field. For higher, $n$ th-order perturbations the problem becomes even worse because, apart from the background quantities, all of the $m$ th-order perturbations with $m<n$ must also be of the form that we have commented [7]. In principle, this restricts to a very narrow physical scenario the possibilities that are left.

Other forms of gauge invariance can be defined using subgroups of $\mathcal{G}$ or ${ }^{\{n\}} \mathcal{G}$. For example invariance with respect to the reduced subgroup of pure $n$ th-order transformations has been used in the past [10], by fixing the gauge perturbatively at all orders $1, \ldots, n-1$, but not at order $n$.

Only perturbations of highly symmetric backgrounds admit a complete description in terms of IGI variables. Even at first order, significant limitations have been found: Stewart and Walker showed that, for vacuum spacetimes, only backgrounds with Petrov type D are possible [6], which fortunately includes the Kerr spacetime. In cosmology, only perturbations of static Friedman-RobertsonWalker (FRW) backgrounds can be described in terms of IGI variables [11]. For spherical backgrounds with matter, only first-order perturbations with axial polarity admit such a description $[12,13]$, but not the complementary set of polar perturbations. This latter result is specially relevant for us, because we want to construct high-order gauge invariants of a spherical spacetime and in Paper I we saw that the polarities mix already at second order: hence there is no hope of getting a purely IGI description in general. Note that vacuum [5] and electrovacuum [14] spacetimes with spherical symmetry are very special cases (in particular included in the cited result for type D spacetimes), for which the programme of construction of gauge invariants can be further developed [15].

On the other hand, when describing gravitational radiation in a vacuum, the Weyl tensor provides all the relevant geometrical information, and therefore many investigations employ it as the basic object to be perturbed. Furthermore, the Weyl tensor defines a set of principal null directions, so that it becomes natural to decompose it using the Newman-Penrose formalism. The analysis of IGI is then simplified, but an additional type of gauge invariance is introduced, called tetrad gauge invariance, which requires invariance under (the 6-parametric Lorentz group of) transformations among null tetrads $[6,13,16]$. We will not use this approach in this paper, but instead appeal to a different and more general notion of gauge invariance in which one makes use of an additional geometrical structure: a privileged gauge $\bar{\phi}_{\varepsilon}$. The basic idea is that, given a family of tensors $\Omega_{\varepsilon}$, one can select a privileged gauge to extract the physical information contained in this family and express this information in terms of the pullback of $\Omega_{\varepsilon}$ in an arbitrary gauge. In other words, the gauge invariant is defined as the function(al) that provides the value of $\bar{\phi}_{\varepsilon}^{*} \Omega_{\varepsilon}$ in a generic gauge:

$$
\bar{\phi}_{\varepsilon}^{*} \Omega_{\varepsilon}=F\left[\phi_{\varepsilon}^{*} \Omega_{\varepsilon}\right] .
$$

So, the gauge invariant is now supplied by the function(al) $F$ rather than by the family of tensors $\Omega_{\varepsilon}$ itself, as was the case for IGI. 
This notion of gauge invariants is similar to that of the constants of motion defined in mechanics by the particular values that the variables of the system take at some fixed instant of time [17], or even to the notion of evolving constants of motion recently introduced in quantum gravity [18] (although in that case one ought to consider a family of privileged gauges parametrized by a set of real numbers, rather than just one of them $\bar{\phi}_{\varepsilon}$ ). In spite of appearing counterintuitive at first, this notion can be very useful in those cases in which the computations can be carried out to completion, i.e. when one can obtain the explicit expression of the invariants in terms of gauge-dependent quantities, in our context, or in terms of time-dependent variables, in mechanics. In other words, we need to determine the explicit form of the gauge transformation $F=$ $\chi_{\varepsilon}^{*}=\phi_{\varepsilon}^{-1} \circ \bar{\phi}_{\varepsilon}$ for arbitrary $\phi_{\varepsilon}$. Whether this is possible or not essentially depends on the choice of gauge $\bar{\phi}_{\varepsilon}$.

In practice, the privileged gauge is defined by imposing some conditions $R_{\varepsilon}$ on the pullback $\bar{\phi}_{\varepsilon}^{*} \tilde{\Omega}_{\varepsilon}$ of a particular tensor $\tilde{\Omega}_{\varepsilon}$. Therefore, $\bar{\phi}_{\varepsilon}$ will be characterized as the gauge in which the tensor $\bar{\phi}_{\varepsilon}^{*} \tilde{\Omega}_{\varepsilon}$ satisfies some specific requirements. For this method to work satisfactorily, this privileged gauge choice has to be rigid. This means that the conditions $R_{\varepsilon}\left[\bar{\phi}_{\varepsilon}^{*} \tilde{\Omega}_{\varepsilon}\right]=0$ must fix uniquely the gauge $\bar{\phi}_{\varepsilon}$, and so any further gauge transformation will violate those conditions.

In perturbation theory, the invariants will then be the combinations ${ }^{\{n\}} F\left[\left\{\Delta_{\phi}^{m}[\Omega]\right\}\right]$ obtained by performing a gauge transformation from the perturbations defined on a generic gauge $\Delta_{\phi}^{m}[\Omega]$ to those defined in the rigid gauge $\Delta_{\bar{\phi}}^{m}[\Omega]$. This kind of combination of perturbations have been characteristic of this approach to gauge invariance, starting with the pioneering work of Moncrief [5] for nonspherical perturbations of Schwarzschild, where the Regge-Wheeler (RW) gauge was implicitly used as the privileged gauge. His work was later generalized by GS [2] to nonspherical perturbations of any spherical background, also implicitly using the RW gauge. The same procedure has been employed by Bardeen [19], Stewart [11], and many other authors in their study of perturbations of FRW cosmologies. It can also be found in several recent investigations of second-order perturbations of vacuum $[8,16,20]$ or cosmological backgrounds [21].

For instance, the first-order gauge invariants of a generic tensor $\Omega$ will be given by

$$
F\left[\Delta_{\phi}[\Omega]\right] \equiv \Delta_{\phi}[\Omega]+\mathcal{L}_{p} \Omega,
$$

where $p^{\mu}$ is the vector field generating the first-order gauge transformation from $\phi$ to $\bar{\phi}$, so that this vector contains now information about our choice of privileged gauge $\bar{\phi}$. Again, in practical applications the gauge $\bar{\phi}$ is selected by imposing some rigid conditions $R$ on the perturbations $\Delta_{\bar{\phi}}[\tilde{\Omega}]$ for some specific tensor $\tilde{\Omega}$, and such that no residual freedom is left in the choice of gauge:

$$
R\left[\Delta_{\bar{\phi}}[\tilde{\Omega}]\right]=0 .
$$

In this way we get the equations

$$
R\left[\Delta_{\phi}[\tilde{\Omega}]+\mathcal{L}_{p} \tilde{\Omega}\right]=0
$$

which must be solved for $p^{\mu}$ in terms of $\Delta_{\phi}[\tilde{\Omega}]$. Substituting the vector $p^{\mu}$ obtained in this way, expressions (14) provide gauge invariants by construction. Note that when $\Omega=\tilde{\Omega}$, some of those expressions (or combinations of them) are trivial identities [equivalent to the requirements (16)]. This method for the determination of invariants can be straightforwardly generalized to higher perturbative orders, as we will see in the following case.

Since metric perturbations play a central role in our analysis, we choose the background metric $g_{\mu \nu}$ as the tensor $\tilde{\Omega}$ on which one imposes the conditions to fix the privileged gauge. We introduce the following compact notation for the perturbations of the metric:

$$
\begin{gathered}
{ }^{\{n\}} h_{\mu \nu} \equiv \Delta_{\phi}^{n}\left[g_{\mu \nu}\right], \\
{ }^{\{n\}} \mathcal{K}_{\mu \nu} \equiv \Delta_{\bar{\phi}}^{n}\left[g_{\mu \nu}\right],
\end{gathered}
$$

for a generic gauge $\phi$ and our privileged one $\bar{\phi}$, respectively. At first order we have that expressions (14) for the metric become

$$
\mathcal{K}_{\mu \nu} \equiv h_{\mu \nu}+\mathcal{L}_{p} g_{\mu \nu} .
$$

The vector $p^{\mu}$ is determined by demanding some conditions $R\left[\mathcal{K}_{\mu \nu}\right]=0$ which characterize the gauge $\bar{\phi}$ at first order. Then, the vector $p^{\mu}$ is determined in terms of the components of $h_{\mu \nu}$ by solving the equations

$$
R\left[h_{\mu \nu}+\mathcal{L}_{p} g_{\mu \nu}\right]=0 .
$$

This completes the definition (19) of the gauge invariant $\mathcal{K}_{\mu \nu}$ as a function of $h_{\mu \nu}$.

Nonetheless we note that, owing to the presence of the Lie derivative, Eqs. (20) contain derivatives of the vector $p^{\mu}$, so that their solution will involve in general integrals of the metric perturbations. Only when $p^{\mu}$ can be determined explicitly in an amenable way from the metric perturbations will we have a useful form of gauge invariants. This fact will depend on the choice of the privileged gauge. In particular, we will see later that around spherical backgrounds the requirement of getting explicit and nonintegral expressions for the harmonic components of the vector $p^{\mu}$ will almost uniquely single out the RW gauge. We also point out that the same vector $p^{\mu}$, obtained by solving Eqs. (20), can now be employed to define the gauge invariants associated with any other tensor $\Omega$ as in Eq. (14). In addition, note that we can still interpret $\mathcal{K}_{\mu \nu}$ as (the value of) the metric perturbations expressed in the rigid gauge $\bar{\phi}$ which satisfies conditions (20). 
At higher orders, and once a rigid gauge is chosen via some conditions ${ }^{\{m\}} R$ for all $m \leq n$, one can obtain the $n$ thorder metric invariants as

$$
{ }^{\{n\}} \mathcal{K}_{\mu \nu} \equiv\{n\} h_{\mu \nu}+\mathcal{L}_{\{n\}} g_{\mu \nu}+{ }^{\{n\}} \mathcal{H}_{\mu \nu} .
$$

Since this equality reflects the effect of a gauge transformation, the source ${ }^{\{n\}} \mathcal{H}_{\mu \nu}$ is explicitly given by Eq. (8) and depends on lower-order vectors ${ }^{\{m\}} p^{\mu}$ and perturbations ${ }^{\{m\}} h_{\mu \nu}$ with $m<n$, but not on ${ }^{\{n\}} p^{\mu}$. Besides, we remember that the source vanishes at first $\operatorname{order}\left({ }^{\{1\}} \mathcal{H}_{\mu \nu}=0\right)$. On the other hand, the equation that one has to solve iteratively in order to determine the gauge vectors ${ }^{\{m\}} p^{\mu}$, from $m=1$ to $m=n$, takes now the expression

$$
{ }^{\{m\}} R\left[{ }^{\{m\}} h_{\mu \nu}+\mathcal{L}_{\{m\}} g_{\mu \nu}+{ }^{\{m\}} \mathcal{H} \mathcal{H}_{\mu \nu}\right]=0 .
$$

In particular, when all the conditions ${ }^{\{m\}} R$ have the same linear functional dependence on their arguments (for instance because they arise from the perturbative expansion of just one set of exact linear gauge conditions on the metric), Eq. (22) will have the form (20) but with the source term ${ }^{\{m\}} R\left[{ }^{\{m\}} \mathcal{H}_{\mu \nu}\right]$. Therefore, the solutions of these equations will be constructed essentially in the same way.

Nakamura has suggested a similar approach [22] to construct high-order gauge invariants. He starts from the basic assumption that a splitting equivalent to Eq. (21) is given from the outset, separating the metric perturbation ${ }^{\{n\}} h_{\mu \nu}$ into its gauge-invariant part ${ }^{\{n\}} \mathcal{K}_{\mu \nu}$ and gaugevariant part (containing the vectors ${ }^{\{m\}} p^{\mu}$ ), with the vectors ${ }^{\{m\}} p^{\mu}$ satisfying some set of requirements. No proposal is made, however, on how such a splitting can be attained. Our scheme goes beyond that proposal, giving a constructive and general prescription to generate the vectors ${ }^{\{m\}} p^{\mu}$ from the choice of a rigid gauge, in such a way that the requirements imposed on ${ }^{\{m\}} p^{\mu}$ are automatically fulfilled.

After determining the vectors $\left\{{ }^{\{1\}} p^{\mu}(h), \ldots,{ }^{\{n\}} p^{\mu}(h)\right\}$, the perturbations of any tensor field, and, in particular, those of the stress-energy tensor ${ }^{\{n\}} \Psi_{\mu \nu}$, can be taken to its gauge-invariant form ${ }^{\{n\}} T_{\mu \nu}$ just by applying a gauge transformation parametrized by the above vectors:

$$
\begin{aligned}
{ }^{\{n\}} T_{\mu \nu}= & \sum_{m=0}^{n} \frac{n !}{(n-m) !} \sum_{\left(K_{m}\right)} \frac{1}{2^{k_{2}} \ldots(m !)^{k_{m}} k_{1} ! \ldots k_{m} !} \\
& \times \mathcal{L}^{k_{1}{ }_{\{1\}} \ldots \mathcal{L}^{k_{m}}{ }_{\{m\}}{ }^{\{n-m\}} \Psi_{\mu \nu} .}
\end{aligned}
$$

In this way we will get a tensor ${ }^{\{n\}} T_{\mu \nu}(\Psi, h)$ whose dependence on the perturbations ${ }^{\{m\}} \Psi_{\mu \nu}$ and ${ }^{\{m\}} h_{\mu \nu}(m \leq n)$ will not change when any gauge transformation is applied to them. In Sec. V we will use these techniques to compute the metric and matter gauge invariants for perturbations of a spherical background spacetime.

\section{SPHERICALLY SYMMETRIC SPACETIMES}

In the following we will restrict our discussion to spherically symmetric backgrounds and employ the notation introduced by GS [2]. We decompose the background manifold $\mathcal{M}$ as the product $\mathcal{M}^{2} \times S^{2}$, where $\mathcal{M}^{2}$ is a two-dimensional Lorentzian manifold and $S^{2}$ is the twosphere. Without loss of generality, any spherically symmetric metric and stress-energy tensor can be written in the block-diagonal form [2]:

$$
\begin{aligned}
g_{\mu \nu}\left(x^{D}, x^{d}\right) d x^{\mu} d x^{\nu}= & g_{A B}\left(x^{D}\right) d x^{A} d x^{B} \\
& +r^{2}\left(x^{D}\right) \gamma_{a b}\left(x^{d}\right) d x^{a} d x^{b}, \\
t_{\mu \nu}\left(x^{D}, x^{d}\right) d x^{\mu} d x^{\nu}= & t_{A B}\left(x^{D}\right) d x^{A} d x^{B} \\
& +\frac{1}{2} r^{2}\left(x^{D}\right) Q\left(x^{D}\right) \gamma_{a b}\left(x^{d}\right) d x^{a} d x^{b},
\end{aligned}
$$

where Greek indices, capital Latin indices, and lowercase Latin indices take values in the ranges $\{0,1,2,3\},\{0,1\}$, and $\{2,3\}$, respectively. In this way, $g_{A B}$ is the metric of the manifold $\mathcal{M}^{2}$, whereas $\gamma_{a b}$ is the round metric on the sphere, and $r$ is a scalar function defined on $\mathcal{M}^{2}$. In order to avoid working explicitly with logarithms of the function $r$, it is usual to define

$$
v_{A} \equiv \frac{r_{, A}}{r}=(\ln r)_{, A} .
$$

We will use the following notation for the covariant derivatives associated with the different introduced metrics:

$$
g_{\mu \nu ; \rho}=0, \quad g_{A B \mid D}=0, \quad \gamma_{a b: d}=0 .
$$

In Paper I we generalized the so-called Regge-WheelerZerilli basis of harmonics [3,23] to any rank. Specifically, we introduced the following two independent rank $s$ symmetric and traceless tensors on the sphere

$$
\begin{aligned}
Z_{l a_{1} \ldots a_{s}}^{m} & \equiv\left(Y_{l: a_{1} \ldots a_{s}}^{m}\right)^{\mathrm{STF}}, \\
X_{l a_{1} \ldots a_{s}}^{m} & \left.\equiv \epsilon_{\left(a_{1}\right.}{ }^{b} Z_{l}^{m} b a_{2} \ldots a_{s}\right)
\end{aligned}
$$

where $Y_{l}^{m}$ are the scalar harmonics and the superscript STF means the symmetric and tracefree part. This definition is valid for $-l \leq m \leq l$ and $1 \leq s \leq l$. In all other cases, these harmonics are defined to be identically zero, except for $s=0$, when $Z_{l}^{m} \equiv Y_{l}^{m}$. These tensor harmonics provide two families with distinct polarity: $Z_{l}^{m} a_{1} \ldots a_{s}$ is polar, whereas $X_{l \quad a_{1} \ldots a_{s}}^{m}$ is axial. Under a parity transformation, the polar and axial harmonics change sign as $(-1)^{l}$ and $(-1)^{l+1}$, respectively.

A basis for tensors of rank $s$ on the sphere is then formed by the two tensors $\left\{Z_{l a_{1} \ldots a_{s}}^{m}, X_{l}^{m} a_{1} \ldots a_{s}\right\}$ and some linear combinations of products between a basis of tensors of rank $(s-2)$, on the one hand, and the metric $\gamma_{a b}$ and the Levi-Civita tensor on the sphere $\epsilon_{a b}$, on the other hand. 


\section{GAUGE-INVARIANT PERTURBATIONS OF A SPHERICAL BACKGROUND}

\section{A. Harmonic decompositions}

When working on spherical backgrounds, it is convenient to decompose our geometrical objects in a series of spherical harmonics in order to remove all angle dependencies from the equations of the problem. We therefore adopt from now on the following decomposition for the different tensors of interest:

$$
\begin{aligned}
& { }^{\{n\}} h_{\mu \nu} \equiv \sum_{l, m}\left(\begin{array}{cc}
{ }^{\{n\}} H_{l A B}^{m} Z_{l}^{m} & { }^{\{n\}} H_{l A}^{m} Z_{l b}^{m}+{ }^{\{n\}} h_{l A}^{m} X_{l b}^{m}{ }^{m}{ }^{m} K_{l}^{m} r^{2} \gamma_{a b} Z_{l}^{m}+{ }^{\{n\}} G_{l}^{m} r^{2} Z_{l a b}^{m}+{ }^{\{n\}} h_{l}^{m} X_{l}^{m} a b
\end{array}\right)
\end{aligned}
$$

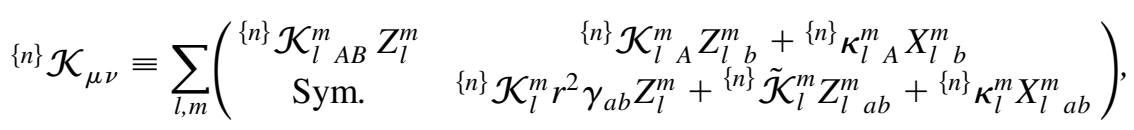

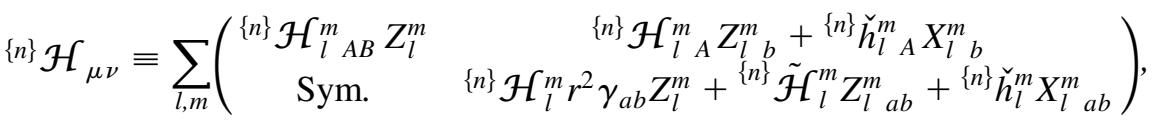

$$
\begin{aligned}
& { }^{\{n\}} p_{\mu} d x^{\mu} \equiv \sum_{l, m}\left[{ }^{\{n\}} P_{l A}^{m} Z_{l}^{m} d x^{A}+r^{2}\left({ }^{\{n\}} P_{l}^{m} Z_{l a}^{m}+{ }^{\{n\}} q_{l}^{m} X_{l a}^{m}\right) d x^{a}\right] .
\end{aligned}
$$

The notation "Sym." indicates that the considered matrices are symmetric. In the most general case, the label $l$ runs over all non-negative integers, whereas $m$ has the usual restriction $-l \leq m \leq l$. Note also that we have adopted the convention that polar (axial) harmonic coefficients are denoted by capital (lowercase) letters.

\section{B. Privileged gauge}

Using the previous harmonic expansions we can decompose the $n$ th-order gauge transformation (21) from the perturbations of the metric in a generic gauge ${ }^{\{n\}} h_{\mu \nu}$ to the perturbations ${ }^{\{n\}} \mathcal{K}_{\mu \nu}$ in some privileged gauge, that we still have to specify:

$$
\begin{aligned}
l \geq 0:{ }^{\{n\}} \mathcal{K}_{l A B}^{m}= & { }^{\{n\}} H_{l A B}^{m}+{ }^{\{n\}} P_{l A \mid B}^{m}+{ }^{\{n\}} P_{l B \mid A}^{m} \\
& +{ }^{\{n\}} \mathcal{H}_{l A B}^{m}, \\
{ }^{\{n\}} \mathcal{K}_{l}^{m}={ }^{\{n\}} K_{l}^{m} & +2 v^{A\{n\}} P_{l A}^{m}-l(l+1)^{\{n\}} P_{l}^{m}+{ }^{\{n\}} \mathcal{H}_{l}^{m},
\end{aligned}
$$

$$
\begin{aligned}
& l \geq 1:{ }^{\{n\}} \mathcal{K}_{l A}^{m}={ }^{\{n\}} H_{l A}^{m}+{ }^{\{n\}} P_{l A}^{m}+r^{2\{n\}} P_{l \mid A}^{m} \\
&+{ }^{\{n\}} \mathcal{H}_{l A}^{m}, \\
&{ }^{\{n\}} \kappa_{l A}^{m}={ }^{\{n\}} h_{l A}^{m}+r^{2\{n\}} q_{l \mid A}^{m}+{ }^{\{n\}} \breve{h}_{l A}^{m}, \\
& l \geq 2:{ }^{\{n\}} \tilde{\mathcal{K}}_{l}^{m}={ }^{\{n\}} G_{l}^{m}+2^{\{n\}} P_{l}^{m}+\frac{1}{r^{2}}{ }^{\{n\}} \tilde{\mathcal{H}}_{l}^{m}, \\
&{ }^{\{n\}} \kappa_{l}^{m}={ }^{\{n\}} h_{l}^{m}+2 r^{2\{n\}} q_{l}^{m}+{ }^{\{n\}} \check{h}_{l}^{m} .
\end{aligned}
$$

The cases $l=0,1$ are special inasmuch as some of the tensor spherical harmonics vanish for these values of $l$, and hence some of the relations that one obtains for $l \geq 2$ are trivially void in these cases.

In general, the privileged gauge at order $n$ will be determined by four conditions ${ }^{\{n\}} R$, imposed on the ten components of ${ }^{\{n\}} \mathcal{K}_{\mu \nu}$. These conditions must rigidly fix the gauge vector (33) as a function(al) of the generic metric perturbation ${ }^{\{n\}} h_{\mu \nu}$. In order to obtain amenable gauge invariants, we would like to select a gauge such that this functional dependence is local with respect to the Lorentzian manifold $\mathcal{M}^{2}$ (whereas the dependence on $S^{2}$ is determined by means of the expansion in tensor harmonics). After replacing the obtained gauge vector, the other six components of ${ }^{\{n\}} \mathcal{K}_{\mu \nu}$ will provide then local (in $\mathcal{M}^{2}$ ) gauge invariants by construction. For simplicity, we will employ from now on the adjective "local" exclusively in the sense of locality in $\mathcal{M}^{2}$, so that no integration over this manifold is involved.

From the above expressions, it is not difficult to see that there are essentially two procedures to end with a vector ${ }^{\{n\}} p^{\mu}$ whose components present a polynomic dependence on those of ${ }^{\{n\}} \mathcal{K}_{\mu \nu}$ and ${ }^{\{m\}} h_{\mu \nu}$, for $m \leq n$. In both cases, the components ${ }^{\{n\}} P_{l}^{m}$ and ${ }^{\{n\}} q_{l}^{m}$ are found solving Eqs. (38) and (39), respectively. But for the vector component ${ }^{\{n\}} P_{l_{A}}^{m}$ one has two options. One can fully determine it from Eq. (36), or one can instead obtain the projection $v^{A\{n\}} P_{l A}^{m}$ from Eq. (35) and the remaining projection $t^{A\{n\}} P_{l A}^{m}$ from Eq. (36), with $t^{A}$ being any vector transverse to $v^{A}$.

This fact suggests two possible sets of conditions to select our privileged gauge, both of them imposed on the tensor ${ }^{\{n\}} \mathcal{K}_{\mu \nu}$. The first one consists of the following 
requirements

$$
\begin{gathered}
l \geq 1:{ }^{\{n\}} \mathcal{K}_{l A}^{m}=0, \\
l \geq 2:{ }^{\{n\}} \tilde{\mathcal{K}}_{l}^{m}=0, \quad{ }^{\{n\}} \kappa_{l}^{m}=0 .
\end{gathered}
$$

This is the RW gauge used at first order [3]. This gauge has been extensively employed in the study of perturbations of spherical backgrounds and leads to a full metric whose pullback satisfies [1]

$$
\left(\bar{\phi}_{\varepsilon}^{*} g_{\varepsilon}\right)_{A b: c} g^{b c}=0, \quad\left(\bar{\phi}_{\varepsilon}^{*} g_{\varepsilon}\right)_{a b}=\tilde{K}_{\varepsilon} g_{a b},
$$

for some generic scalar field $\tilde{K}_{\varepsilon}$ defined in the background four-dimensional manifold $\mathcal{M}$. It is well known that this gauge is not completely rigid because it does not impose any restriction on some of the $l=0,1$ components of the metric perturbations, at any order. For the rest of this subsection, we will restrict our analysis of gauge invariance to transformations whose generators do not contain the corresponding $l=0,1$ modes (both at order $n$ and lower orders). We defer the analysis of the general case to the next subsection.

With the choice of the RW gauge, Eqs. (36), (38), and (39) are the conditions that must be solved to obtain the gauge vector ${ }^{\{n\}} p^{\mu}$, which turns out to be

$$
\begin{gathered}
{ }^{\{n\}} P_{l A}^{m}=\frac{r^{2}}{2}\left({ }^{\{n\}} G_{l}^{m}+\frac{1}{r^{2}}{ }^{\{n\}} \tilde{\mathcal{H}}_{l}^{m}\right)_{\mid A}-{ }^{\{n\}} H_{l A}^{m}-{ }^{\{n\}} \mathcal{H}_{l A}^{m}, \\
\{n\} P_{l}^{m}=-\frac{1}{2}\left({ }^{\{n\}} G_{l}^{m}+\frac{1}{r^{2}}{ }^{\{n\}} \tilde{\mathcal{H}}_{l}^{m}\right), \\
{ }^{\{n\}} q_{l}^{m}=-\frac{1}{2 r^{2}}\left({ }^{\{n\}} h_{l}^{m}+{ }^{\{n\}} \check{h}_{l}^{m}\right) .
\end{gathered}
$$

Substituting these expressions in Eqs. (34), (35), and (37) we obtain explicitly the high-order metric gauge invariants.

The axial part of the second algebraic gauge is equivalent to the RW gauge, and therefore ${ }^{\{n\}} q_{l}^{m}$ is given again by Eq. (45). As a consequence, the axial invariant is the same as before, namely ${ }^{\{n\}} \kappa_{l A}^{m}$. However, the polar part is now determined by the following conditions:

$$
\begin{gathered}
l \geq 0:{ }^{\{n\}} \mathcal{K}_{l}^{m}=0, \\
l \geq 1:{ }^{\{n\}} \mathcal{K}_{l{ }^{m}}^{m} t^{A}=0, \\
l \geq 2:{ }^{\{n\}} \tilde{\mathcal{K}}_{l}^{m}=0,
\end{gathered}
$$

which generalize the Campolattaro-Thorne gauge $K=0$ [24] for the $l=1$ component of first-order perturbations. The exact gauge would be

$$
\left(\bar{\phi}_{\varepsilon}^{*} g_{\varepsilon}\right)_{A b: c} g^{b c}=\epsilon_{A B} t^{B} \tilde{H}_{\varepsilon}, \quad\left(\bar{\phi}_{\varepsilon}^{*} g_{\varepsilon}\right)_{a b}=g_{a b},
$$

for some scalar field $\tilde{H}_{\varepsilon}$ in the background $\mathcal{M}$. The above comments about the lack of rigidity are also applicable to this gauge. That is, the gauge generators are assumed to have no component with harmonic label $l=0,1$.

For the polar components of the vector ${ }^{\{n\}} p^{\mu}$, we solve conditions (46) and obtain

$$
\begin{aligned}
& v^{A} P_{l A}^{m}=-\frac{l(l+1)}{4}\left({ }^{\{n\}} G_{l}^{m}+\frac{1}{r^{2}}{ }^{\{n\}} \tilde{\mathcal{H}}_{l}^{m}\right)-\frac{1}{2}{ }^{\{n\}} K_{l}^{m}, \\
& t^{A} P_{l A}^{m}=\frac{r^{2}}{2} t^{A}\left({ }^{\{n\}} G_{l}^{m}+\frac{1}{r^{2}}{ }^{\{n\}} \tilde{\mathcal{H}}_{l}^{m}\right)_{\mid A} \\
& -t^{A}\left({ }^{\{n\}} H_{l A}^{m}+{ }^{\{n\}} \mathcal{H}_{l A}^{m}\right), \\
& P_{l}^{m}=-\frac{1}{2}\left({ }^{\{n\}} G_{l}^{m}+\frac{1}{r^{2}}{ }^{\{n\}} \tilde{\mathcal{H}}_{l}^{m}\right) .
\end{aligned}
$$

Substituting the above relations in the respective definitions (34) and (36), one arrives at the corresponding polar invariants $\mathcal{K}_{l A B}^{m}$ and $\boldsymbol{v}^{A} \mathcal{K}_{l A}^{m}$.

In conclusion, we have seen that there are two choices of gauge that lead to a polynomic and local expression of the gauge vector ${ }^{\{n\}} p^{\mu}$ in terms of the metric perturbation ${ }^{\{n\}} h_{\mu \nu}$ in an arbitrary gauge. For both of them, one obtains a set of local gauge invariants, under the restriction $l \geq 2$ on the modes of the gauge generators, at all perturbative orders. These two sets are inequivalent. Nevertheless, the RW gauge does not need the introduction by hand of a vector field $t_{A}$ transverse to $v_{A}$, so that one can consider it to be more natural, at least as far as this kind of geometric considerations are concerned. Because of these reasons, we choose the RW gauge as our privileged gauge from now on. In Paper I we already computed the evolution equations for the perturbations in the RW gauge. Of course, those equations are also valid for the gauge invariants that are tied to this gauge.

The fact that there exist privileged gauges that provide local gauge invariants is related to the spherical symmetry of the background, which allows the choice of an adapted system of coordinates in which the metric has a blockdiagonal form. Besides, Kodama, Ishibashi, and Seto [25] have shown that it is possible to generalize the first-order GS invariants for any metric which can be written in blockdiagonal form in an $(m+n)$-dimensional manifold $\mathcal{M}^{m} \times$ $\mathcal{K}^{n}$ with a maximally symmetric block in $\mathcal{K}^{n}$. This means that a higher-dimensional form of the RW gauge is always attainable, though the corresponding conditions on the metric perturbations may be different.

\section{The particular cases $l=0,1$}

It is well known that, at first order, one cannot construct local gauge invariants for $l=0,1$ by the methods explained in the previous subsection. This is because some of the Eqs. (34)-(39) are not present in those cases, and therefore it is not possible to attain a local expression for 
the components of the gauge vector ${ }^{\{n\}} p^{\mu}$ in terms of those of the metric perturbations. Of course, gauge conditions different from the RW ones can be imposed on the metric perturbations and hence one can obtain from them the associated gauge invariants, but these will be nonlocal because the gauge vector will be given by an integral expression (over $\mathcal{M}^{2}$ ) of the metric perturbations. Whether this is useful or not will depend on the particular application that one is studying.

The same obstruction appears as well at every order in perturbation theory, so that one cannot get local gauge invariants for $l=0,1$ at any order. However, mode coupling makes the problem worse: the existence of lowerorder modes with $l=0,1$ may prevent the construction of higher-order local gauge invariants with $l \geq 2$. This will happen when such lower-order modes have a nonzero contribution to the sources $\mathcal{H}$ of Eqs. (34)-(39).

The restriction on the gauge generators introduced in the previous subsection is a valid alternative for first-order perturbation theory: the GS gauge invariants remain unchanged under the restricted group ${ }^{\{1\}} \mathcal{G}^{\diamond}$, where the diamond indicates that no first-order generator with $l=0,1$ is included. However, removing all $l=0,1$ generators is already inconsistent at second order if we demand invariance under a group of transformations, because the $l=0,1$ components of the vectors ${ }^{\{2\}} \xi$ will be unavoidably generated by coupling of first-order gauge modes [cf. composition (12)]. Fortunately, those offending gauge modes act only on the $l=0,1$ second-order perturbations, for which invariants cannot be constructed anyway. All other secondorder perturbations admit a gauge-invariant form, as given in the previous subsection, under the gauge group ${ }^{\{2\}} G^{\diamond}$ where again the diamond denotes that no first-order $l=0$, 1 gauge mode is included, but all other first and secondorder (including $l=0,1)$ modes are allowed. That is one of the main results of this investigation: gauge-invariant perturbations can be consistently and simultaneously constructed for all $l \geq 2$ modes at second order, all being invariant under the group ${ }^{\{2\}} \mathcal{G}^{\diamond}$.

The situation at third and higher orders is more restrictive. As a summary, working at order $n>2$ only a finite number of gauge generators can be included in the invariance group at orders $1, \ldots, n-2$. The unavoidable presence of second-order gauge modes with $l=0,1$ couples to any metric perturbation at order $m$ and with harmonic label $l$, preventing the construction of a gauge invariant of order $m+2$ and label $l$ or $l \pm 1$, with respect to those gauge modes. The detailed analysis is shown in Appendix A.

\section{First-order gauge invariants}

We now particularize our discussion to first-order perturbations and reproduce the GS first-order gauge invariants [2], which are a generalization of the invariants introduced by Moncrief [5] for a Schwarzschild background.

Since the source ${ }^{\{1\}} \mathcal{H}_{\mu \nu}$ vanishes, from Eqs. (34), (35), and (37) one gets the metric invariants

$$
\begin{gathered}
{ }^{\{1\}} \mathcal{K}_{l_{A B}}^{m}={ }^{\{1\}} H_{l_{A B}}^{m}+{ }^{\{1\}} P_{l_{A \mid B}^{m}}+{ }^{\{1\}} P_{l_{B \mid A}^{m}}, \\
{ }^{\{1\}} \mathcal{K}_{l}^{m}={ }^{\{1\}} K_{l}^{m}+2 v^{A\{1\}} P_{l_{A}}^{m}-l(l+1)^{\{1\}} P_{l}^{m}, \\
\{1\} \kappa_{l_{A}}^{m}={ }^{\{1\}} h_{l_{A}}^{m}+r^{2\{1\}} q_{l_{\mid A}}^{m},
\end{gathered}
$$

where

$$
\begin{gathered}
{ }^{\{1\}} P_{l A}^{m}=\frac{r^{2}}{2}{ }^{\{1\}} G_{l \mid A}^{m}, \\
{ }^{\{1\}} P_{l}^{m}=-\frac{1}{2}{ }_{\{1\}} G_{l}^{m}, \\
{ }^{\{1\}} q_{l}^{m}=-\frac{1}{2 r^{2}}{ }^{\{1\}} h_{l}^{m} .
\end{gathered}
$$

Using these expressions for the gauge vector, it is possible to construct the first-order gauge invariants corresponding to any other (matter or metric) tensorial object.

\section{E. Second-order gauge invariants}

The second-order metric invariants can be obtained by substituting expressions (43)-(45) in Eqs. (34), (35), and (37) for $n=2$, whereas the matter invariants, constructed from the perturbations ${ }^{\{n\}} \Psi_{\mu \nu} \equiv \Delta^{n}\left[t_{\mu \nu}\right]$, are encoded in the tensor ${ }^{\{2\}} T_{\mu \nu}$ defined by

$$
\begin{aligned}
{ }^{\{2\}} T_{\mu \nu}= & { }^{\{2\}} \Psi_{\mu \nu}+\mathcal{L}_{\{2\}} \Psi_{\mu \nu}+\mathcal{L}_{\{1\}}^{2} \Psi_{\mu \nu} \\
& +2 \mathcal{L}_{\{1\} p}{ }^{\{1\}} \Psi_{\mu \nu} .
\end{aligned}
$$

For the sake of brevity we will only provide explicitly the form of the metric gauge invariants, but not that of the matter invariants, which can be computed along the same lines.

Our aim is to compute the harmonic coefficients (32) of the source

$$
{ }^{\{2\}} \mathcal{H}_{\mu \nu} \equiv \mathcal{L}_{\{1\}}^{2} g_{\mu \nu}+2 \mathcal{L}_{\{1\}}{ }^{\{1\}} h_{\mu \nu} .
$$

These coefficients will have the general form (we obviate the $n=2$ label for simplicity):

$$
\begin{aligned}
\mathcal{H}_{l A B}^{m} & =\sum_{\bar{l}, \hat{l}} \sum_{\bar{m}, \hat{m}}{ }^{(\epsilon)} \mathcal{H}_{\bar{l} \hat{l} l^{m}{ }^{\bar{m}}{ }_{A B},} \\
\mathcal{H}_{l A}^{m} & =\sum_{\bar{l}, \hat{l}} \sum_{\bar{m}, \hat{m}}{ }^{(\epsilon)} \mathcal{H}_{\bar{l} \hat{l} \hat{l}^{l}{ }^{m} A}, \\
\mathcal{H}_{l}^{m} & =\sum_{\bar{l}, \hat{l}} \sum_{\bar{m}, \hat{m}}{ }^{(\epsilon)} \mathcal{H}_{\bar{l} \hat{l} l}^{\bar{m} \hat{m} m},
\end{aligned}
$$




$$
\begin{gathered}
\tilde{\mathcal{H}}_{l}^{m}=\sum_{\bar{l}, \hat{l}} \sum_{\bar{m}, \hat{m}}{ }^{(\epsilon)} \tilde{\mathcal{H}}_{\bar{l} \hat{l} \hat{l} l}^{\bar{m} m}, \\
\check{h}_{l A}^{m}=-i \sum_{\bar{l}, \hat{l}} \sum_{\bar{m}, \hat{m}}{ }^{(-\epsilon)} \mathcal{H}_{\bar{l} \hat{m} \hat{l}^{\prime} l A}, \\
\check{h}_{l}^{m}=-i \sum_{\bar{l}, \hat{l}} \sum_{\bar{m}, \hat{m}}{ }^{(-\epsilon)} \mathcal{H}_{\bar{l} \hat{m} l}^{\bar{m} \hat{m} m},
\end{gathered}
$$

with $\bar{l}$ and $\hat{l}$ being the harmonic labels corresponding to the two first-order modes that get coupled. They are independent and run over all non-negative integers with the restriction $|\bar{l}-\hat{l}| \leq l \leq|\bar{l}+\hat{l}|$. In addition, one must impose the usual restrictions on the values of the labels $\bar{m}$ and $\hat{m}$. We have also defined the alternating sign $\epsilon \equiv$ $(-1)^{\bar{l}+\hat{l}-l}$. As it is shown in Appendix A, this alternating sign encodes the mixing of polarities caused by the product of tensor harmonics in higher-order perturbation theory. For a detailed explanation of this effect and the use of the sign $\epsilon$ we refer the reader to Paper I. As in that reference, all computations have been carried out with the computer algebra system xTensor [26].

From now on and without loss of generality, we will suppose that there are only two first-order modes that will be denoted as $(\hat{\mathcal{K}}, \hat{p})$ and $(\overline{\mathcal{K}}, \bar{p})$. The tensorial sources are given by

$$
\begin{aligned}
& { }^{(+)} \mathcal{H} \mathcal{H}_{\bar{l} \hat{l} l{ }_{A B} \hat{m}}=-4 E_{1 \hat{l} \hat{m} l}^{-1 \bar{l} \bar{P}}\left\{\bar{P} \hat{\mathcal{K}}_{A B}+2 \bar{q}_{\mid(B} \hat{\kappa}_{A)}-\left(\bar{P} \hat{P}_{(B}\right)_{\mid A)}\right. \\
& \left.-r^{2} \hat{P}_{\mid A} \bar{P}_{\mid B}-r^{2} \hat{q}_{\mid A} \bar{q}_{\mid B}\right\} \\
& +2 E_{0 \hat{l} \hat{m} l}^{0 \bar{l} \bar{m}}\left\{2 \bar{P}_{\mid(A}^{C} \hat{\mathcal{K}}_{B) C}+\bar{P}^{C} \hat{\mathcal{K}}_{A B \mid C}\right. \\
& \left.-\hat{P}_{\mid(B}^{C} \bar{P}_{A) \mid C}-\hat{P}_{\mid A}^{C} \bar{P}_{C \mid B}-\hat{P}^{C} \bar{P}_{(A \mid B) C}\right\}, \\
& { }^{(-)} \mathcal{H} \mathcal{H}_{\bar{l} \hat{l} \hat{m} l_{A B}}=-4 i E_{1 \hat{l} \hat{m} l}^{-1 \bar{l} \bar{m}}\left\{\hat{q} \overline{\mathcal{K}}_{A B}+2 \bar{P}_{\mid(A} \hat{\kappa}_{B)}\right. \\
& \left.-2 r^{2} \hat{q}_{\mid(A} \bar{P}_{\mid B)}+\bar{q} \hat{P}_{(A \mid B)}+\bar{q}_{\mid(A} \hat{P}_{B)}\right\} \text {. }
\end{aligned}
$$

The real coefficients $E$ come from the product of harmonics [1] that is implicit in Eq. (60). They are defined as

$$
E_{s^{\prime} l^{\prime} m^{\prime} l^{\prime \prime}}^{l m} \equiv \frac{k\left(l^{\prime},\left|s^{\prime}\right|\right) k(l,|s|)}{k\left(l^{\prime \prime},\left|s+s^{\prime}\right|\right)} C_{l^{\prime} l l^{\prime \prime}}^{m^{\prime} m m^{\prime}+m} C_{l^{\prime} l l^{\prime \prime}}^{s^{\prime} s s^{\prime}+s},
$$

where $C_{l_{1} l_{2} l}^{m_{1} m_{2} m_{1}+m_{2}}$ are Clebsch-Gordan coefficients and we have defined the normalization factor

$$
k(l, s) \equiv \sqrt{\frac{(2 l+1)(l+s) !}{2^{s+2} \pi(l-s) !}} .
$$

The vectorial sources are decomposed as

$$
\begin{aligned}
& { }^{(+)} \mathcal{H}_{\bar{l} \hat{m} l A}^{\bar{m} \hat{l} m}=E_{-1 \hat{l} \hat{m} l}^{2} \bar{l}_{\bar{m}}\left\{-2\left(\bar{q} \hat{\kappa}_{A}+\hat{q} \bar{\kappa}_{A}\right)+\left(\hat{P}_{A} \bar{P}+\bar{P}_{A} \hat{P}\right)+r^{2}\left(3 \bar{P} \hat{P}_{\mid A}+\hat{P} \bar{P}_{\mid A}\right)+2 \bar{q}\left(r^{2} \hat{q}\right)_{\mid A}+r^{6}\left(\frac{\hat{q} \bar{q}}{r^{4}}\right)_{\mid A}\right\} \\
& +\frac{1}{2} E_{1 \hat{l} \hat{m} l}^{0 \bar{l} \bar{l}}\left\{2 \bar{l}(\bar{l}+1)\left(\hat{q} \bar{\kappa}_{A}-\bar{q} \hat{\kappa}_{A}\right)+4 \hat{P}^{B} \overline{\mathcal{K}}_{A B}+4 r^{2} \hat{P}_{\mid A} \overline{\mathcal{K}}+\bar{l}(\bar{l}+1)\left(\bar{P} \hat{P}_{A}+\hat{P} \bar{P}_{A}+3 r^{2} \bar{P} \hat{P}_{\mid A}+r^{2} \hat{P} \bar{P}_{\mid A}\right.\right. \\
& \left.\left.+r^{2} \bar{q} \hat{q}_{\mid A}-r^{2} \hat{q} \bar{q}_{\mid A}\right)-2 r^{2}\left[\left(\bar{P}^{B} \hat{P}_{\mid B}\right)_{\mid A}+4 v^{B} \bar{P}_{B} \hat{P}_{\mid A}\right]-2\left(\bar{P}^{B} \hat{P}_{A \mid B}+2 \hat{P}^{B} \bar{P}_{B \mid A}+\hat{P}^{B} \bar{P}_{A \mid B}\right)\right\}, \\
& { }^{(-)} \mathcal{H} \bar{l}_{\bar{l} \hat{l} \hat{l}_{A} m}^{\bar{l}{ }^{2}}=-i E_{-1 \hat{l} \hat{m} l}^{2 \bar{m} \bar{m}}\left\{2\left(\hat{P} \bar{\kappa}_{A}-\bar{P} \hat{\kappa}_{A}\right)+\hat{q} \bar{P}_{A}-\bar{q} \hat{P}_{A}+r^{2} \hat{q} \bar{P}_{\mid A}+3 r^{2} \bar{P} \hat{q}_{\mid A}-3 r^{2} \bar{q} \hat{P}_{\mid A}-r^{2} \hat{P} \bar{q}_{\mid A}\right\} \\
& +i E_{0 \hat{l} \hat{m} l}^{1 \bar{l} \bar{m}}\left\{2 r^{2} \hat{\mathcal{K}} \bar{q}_{\mid A}-\hat{l}(\hat{l}+1)\left(\hat{P} \bar{\kappa}_{A}+\bar{P} \hat{\kappa}_{A}\right)+2\left(\bar{\kappa}^{B} \hat{P}_{B \mid A}+\hat{P}^{B} \bar{\kappa}_{A \mid B}\right)+\frac{\hat{l}(\hat{l}+1)}{2}\left(r^{2} \bar{q} \hat{P}_{\mid A}-r^{2} \hat{q} \bar{P}_{\mid A}+3 r^{2} \hat{P} \bar{q}_{\mid A}\right.\right. \\
& \left.\left.+r^{2} \bar{P} \hat{q}_{\mid A}-\hat{q} \bar{P}_{A}+\bar{q} \hat{P}_{A}\right)-r^{2}\left(\hat{P}^{B} \bar{q}_{\mid B A}+\hat{P}_{\mid A}^{B} \bar{q}_{\mid B}+4 v^{B} \hat{P}_{B} \bar{q}_{\mid A}\right)\right\},
\end{aligned}
$$

and finally the four scalar sources are given by

$$
\begin{aligned}
{ }^{(+)} \mathcal{H} \mathcal{H}_{\bar{l} \hat{l} l}^{\bar{m} \hat{l} m}= & -4 E_{2 \hat{l} \hat{m} l}^{-2 \bar{l} \bar{q}}(\hat{q} \bar{q}+\hat{P} \bar{P})+E_{1 \hat{l} \hat{m} l}^{-1 \bar{l} \bar{m}}\left\{-4 \hat{P} \overline{\mathcal{K}}-[\hat{l}(\hat{l}+1)+\bar{l}(\bar{l}+1)] \hat{P} \bar{P}+\frac{2}{r^{2}} \hat{P}^{A}\left(r^{2} \bar{P}\right)_{\mid A}+\frac{2}{r^{2}} \hat{P}^{A} \bar{P}{ }_{A}\right\} \\
& +E_{0 \hat{l} \hat{m} l}^{0 \bar{l} \bar{m}}\left\{-2 \bar{l}(\bar{l}+1) \bar{P} \hat{\mathcal{K}}+\frac{2}{r^{2}} \bar{P}^{A}\left(r^{2} \hat{\mathcal{K}}\right)_{\mid A}+\frac{\hat{l}(\hat{l}+1)}{r^{4}} \bar{P}^{A}\left(r^{4} \hat{P}\right)_{\mid A}-\hat{l}(\hat{l}+1) \bar{l}(\bar{l}+1) \hat{P} \bar{P}-2 \hat{P}^{A}\left[\left(\bar{P}^{B} v_{B}\right)_{\mid A}\right.\right. \\
& \left.\left.+2 \bar{P}^{B} v_{A} v_{B}\right]\right\}, \\
& \left(\mathcal{H}_{\bar{l} \hat{l} l}^{\bar{m} \hat{m} m}=8 i E_{2}^{-2 \bar{l} \bar{l} \hat{m} l} \hat{P} \bar{q}-2 i E_{1}^{-1 \hat{l} \bar{m} l}\left\{\frac{2}{r^{2}} \bar{P}^{A} \hat{\kappa}_{A}-2 \bar{q} \hat{\mathcal{K}}-\hat{l}(\hat{l}+1) \hat{P} \bar{q}+\frac{1}{r^{2}} \hat{P}^{A}\left(r^{2} \bar{q}\right)_{\mid A}\right\},\right.
\end{aligned}
$$




$$
\begin{aligned}
{ }^{(+)} \tilde{\mathcal{H}}_{\bar{l} \hat{l} l}^{\bar{m} \hat{m} m}= & 2 r^{2} E_{3}^{-1 \bar{l} \bar{l} \hat{m} l}(\hat{q} \bar{q}+\hat{P} \bar{P})+r^{2} E_{1 \hat{l} \hat{m} l}^{1 \bar{l} \bar{m} l} \\
& \times\left\{(\hat{l}+2)(\hat{l}-1)[\hat{P} \bar{P}-\hat{q} \bar{q}]-2 \hat{P}^{A} \bar{P}_{\mid A}\right. \\
& \left.-\frac{2}{r^{2}} \hat{P}^{A} \bar{P}_{A}\right\}+2 r^{2} E_{2 \bar{l} \hat{l} \hat{m} l}^{0 \bar{l}}\{2 \hat{P} \overline{\mathcal{K}}+\bar{l}(\bar{l}+1) \\
& \left.\times[\hat{q} \bar{q}+2 \hat{P} \bar{P}]-\frac{1}{r^{4}} \bar{P}^{A}\left(r^{4} \hat{P}\right)_{\mid A}\right\}, \\
{ }^{(-)} \tilde{\mathcal{H}}_{\bar{l} \hat{m} l}^{\bar{m} \hat{m} m}= & 2 i r^{2} E_{3}^{-1 \bar{l} \bar{l} \hat{m} l}(\hat{q} \bar{P}-\hat{P} \bar{q})+i E_{2 \hat{l} \hat{m} l}^{0 \bar{l} \bar{m}}\left\{4 r^{2} \hat{q} \overline{\mathcal{K}}\right. \\
& \left.+2 r^{2} \bar{l}(\bar{l}+1)[2 \bar{P} \hat{q}-\hat{P} \bar{q}]-\frac{2}{r^{2}} \bar{P}^{A}\left(r^{4} \hat{q}\right)_{\mid A}\right\} \\
& +i E_{1 \hat{l} \hat{m} l}^{1 \bar{l} \bar{m}}\left\{4 \bar{P}^{A} \hat{\kappa}_{A}+(\hat{l}+2)(\hat{l}-1)\right. \\
& \left.\times r^{2}[\hat{P} \bar{q}+\bar{P} \hat{q}]-2 r^{2} \hat{P}^{A} \bar{q}_{\mid A}\right\} .
\end{aligned}
$$

These formulas allow us to construct the gauge-invariant form (under the restricted group ${ }^{\{2\}} \mathcal{G}^{\diamond}$ of transformations) of any second-order metric perturbation in a covariant way. Note that we have not restricted the harmonic labels corresponding to the first-order metric perturbations. In particular we allow the values $l=0$ and $l=1$ for them, although then some of the harmonic coefficients in the formulas will vanish.

Appendix B studies the particularization of these gauge invariants to the case of second-order perturbations of a vacuum in Schwarzschild coordinates when one has at first order a single polar mode with $l=2$, comparing the results with those of Ref. [8].

\section{CONCLUSIONS}

We have completed the construction of a framework for the analysis of high-order gauge-invariant nonspherical perturbations of a general spherical spacetime, with any type of matter content. Our work started in Paper I by generalizing to higher orders the GS formalism for firstorder perturbations, although the perturbations were described in that work in a particular gauge. In this article we have given explicit algorithms to construct gauge-invariant perturbations at any order, focusing again on the second order, for which we have provided full expressions of the metric gauge invariants.

Before doing that, it has been necessary to discuss in detail what we mean by gauge invariance. The simplest or most natural form of gauge invariance (introduced by Sachs [4]) turns out to be too restrictive in spherical symmetry, even more when dealing with high-order perturbations. We have used an alternative form of gauge invariance, based on the work by Moncrief [5] on perturbations about Schwarzschild. Borrowing ideas from Bardeen [19] and Gleiser et al. [20], we interpret Moncrief's gauge invariants as being associated with a choice of preferred gauge. This reinterpretation has allowed us to extend the construction of gauge invariants to higher orders in perturbation theory, our main goal in this work.

The question then arises of what has been really gained in defining gauge invariants if these are associated with a preferred gauge. First of all, it is important to emphasize that these are true invariants, in the sense that they do not change under gauge transformations of the perturbations, and therefore in a given problem we do not have to worry about the gauge in which the perturbations actually are described. Second, the freedom to choose the preferred gauge is severely restricted in practice by the requirement of obtaining amenable explicit expressions for the invariants. For example in spherical symmetry the demand of arriving at explicit expressions that are local with respect to the dependence on $\mathcal{M}^{2}$ almost singles out the RW gauge, which essentially renders the associated invariants unique. For nonsymmetric background spacetimes this type of gauge invariants, with an explicit and manageable form in terms of the perturbations in a generic gauge, will generically not exist.

Unfortunately the RW gauge cannot be imposed on perturbations with $l=0$ or $l=1$ at all orders, and this obstacle propagates to higher values of $l$ through mode coupling. In this way, the invariants associated with the RW gauge remain unaltered under the set of gauge transformations whose generators exclude all the modes with harmonic label $l$ equal to 0 or 1 . In general, however, this set of transformations does not have group structure owing to mode coupling. Nevertheless, at second order, the group ${ }^{\{2\}} \mathcal{G}^{\diamond}$ (which does not contain the $l=0,1$ first-order gauge generators) allows the construction of all gauge invariants with $l \geq 2$ both at first and second orders, invariants that are exclusively associated with the RW gauge and whose expression we have been able to deduce for the most general case (i.e., for any matter model and any background spacetime).

Our framework is now ready to be applied to specific matter models and we are currently working on its application to the study of second-order perturbations of a timedependent ball of perfect fluid, with a general (2parameter) equation of state [27].

\section{ACKNOWLEDGMENTS}

We thank Vincent Moncrief for helpful conversations. D. B. acknowledges financial support from the FPI program of the Regional Government of Madrid. J. M. M.-G. acknowledges financial aid provided by the I3P framework of CSIC and the European Social Fund. This work was supported by the Spanish MEC Project No. FIS200505736-C03-02.

\section{APPENDIX A: MODE COUPLING AMONG GAUGE GENERATORS}

We have constructed second-order gauge invariants under the group ${ }^{\{2\}} \mathcal{G}^{\diamond}$ starting just from the non(fully-) 
rigid RW gauge. This is not possible at higher orders and actually one has to restrict to a finite set of lower-order modes both in the gauge generators and in the perturbations in order to define some form of gauge invariance. This is because the presence at first order of any gauge mode $l \geq 2$ will generate, just by self-coupling, the second-order modes with harmonic labels 0 and 1 . It will then be impossible to construct the gauge-invariant form of a third-order perturbation whose source $\mathcal{H}$ contains a term coupling any of these second-order $l=0,1$ modes with any first-order mode. But those sources generically contain all possible couplings, and so only a problem in which we restrict the number of first-order gauge modes allows some form of gauge invariance at third order. In this appendix we will analyze generic mode coupling around spherical symmetry, starting at second order and then proceeding to higher orders. We will later give some bounds on the number of modes that can be present at first order to allow for the construction of a $n$ th-order mode with label $l$.

The second-order $l$-mode will get a contribution from a pair of first-order modes $\hat{l}$ and $\bar{l}$ if two conditions are obeyed. On the one hand, the harmonic labels must be related by the usual composition formula

$$
|\hat{l}-\bar{l}| \leq l \leq \hat{l}+\bar{l} .
$$

On the other hand, mode coupling must conserve parity. To any harmonic coefficient with label $l$, we associate a polarity sign $\sigma$ such that, under parity, the harmonic changes by a sign $\sigma(-1)^{l}$. Polar (axial) harmonics have $\sigma=+1(\sigma=$ $-1)$. Then, parity conservation implies the second condition:

$$
(-1)^{\bar{l}+\hat{l}-l} \equiv \epsilon=\sigma \bar{\sigma} \hat{\sigma},
$$

where we have made use of the alternating sign $\epsilon$. There is a special case in which the coupling of two modes satisfying Eqs. (A1) and (A2) does not contribute to a secondorder mode, and the reason comes from the properties of the Clebsch-Gordan coefficients that appear in the product formula for the tensor harmonics (see Paper I). In axisymmetry $(\bar{m}=\hat{m}=0)$ the Clebsch-Gordan coefficients, and as a consequence the $E$ coefficients that couple the modes [defined in Eq. (69)], vanish if $\bar{l}+\hat{l}+l$ is odd. A highly geometric derivation of the perturbative structure under the assumption of axisymmetry is given in Ref. [12].

This analysis can be extended to higher orders. In particular, the parity condition will be that a collection of $k$ modes with harmonic labels $\left\{l_{1}, \ldots, l_{k}\right\}$ and polarities $\left\{\sigma_{1}, \ldots, \sigma_{k}\right\}$ will contribute to the mode $(l, \sigma)$ only if $(-1)^{l} \sigma=\prod_{i=1}^{k}(-1)^{l_{i}} \sigma_{i}$.

Let us finally consider the case in which we have a firstorder finite collection of modes with their harmonic labels taking all the values from $l=2$ to $l=l_{\max }$, with contributions from both the polar and the axial sectors. Coupling of these modes at order $n$ will generate some new modes, following the above rules, so that the highest value of their harmonic label will be $n l_{\max }$. The construction of the gauge invariants under the corresponding group of transformations and tied to the RW gauge is only guaranteed for those modes with harmonic label greater than $(n-2) l_{\max }+1$. This number comes from the coupling of the $(n-2)$ thorder $(n-2) l_{\max }$-mode with the second-order $l=1$ mode.

\section{APPENDIX B: COMPARISON WITH THE RESULTS OF GARAT AND PRICE}

Garat and Price calculated the contribution of two axisymmetric $(\bar{m}=\hat{m}=0)$ first-order polar quadrupoles $(\bar{l}=$ $\hat{l}=2)$ to the $l=2$ multipole of the second-order Zerilli gauge invariant [8]. Their calculation was done in Schwarzschild $(t, r)$ coordinates. In order to translate their coordinate-dependent expressions to our covariant notation, we introduce the following frame of vectors:

$$
r^{A} \equiv f\left(\frac{\partial}{\partial r}\right)^{A}, \quad t^{A} \equiv f^{-1}\left(\frac{\partial}{\partial t}\right)^{A},
$$

where $f \equiv \sqrt{r^{\mid A} r_{\mid A}}=\sqrt{1-2 M / r}$ and $M$ is the background black hole mass. They are normalized in the following way,

$$
r^{A} r_{A}=1, \quad t^{A} t_{A}=-1, \quad r^{A} t_{A}=0 .
$$

The metric and Levi-Civita tensor are given by

$$
\begin{gathered}
g_{A B}=-t_{A} t_{B}+r_{A} r_{B}, \\
\epsilon_{A B}=r_{A} t_{B}-r_{B} t_{A} .
\end{gathered}
$$

Note that the relation between our frame vectors and the coordinate 1 -forms is

$$
r_{A}=f^{-1} r_{\mid A}, \quad t_{A}=-f t_{\mid A} .
$$

Another question to consider in order to compare our results with those of Garat and Price is the definition of the tensor spherical harmonics. First, their normalization is different, which makes their harmonic coefficients to be a factor $\alpha^{-1} \equiv 2 \sqrt{\pi / 5}$ greater than ours. Second, there is a mix of harmonic coefficients $G$ and $K$ because Garat and Price employed a harmonic proportional to $Y_{2: a b}^{0}$ instead of using $Z_{2 a b}^{0}$.

The translation from the harmonic coefficients used by Garat and Price (which appear below in the left-hand side and in their notation) to our coefficients is

$$
\begin{aligned}
& H_{0}^{(n)} \rightarrow \alpha t^{A} t^{B\{n\}} H_{A B}, \\
& H_{1}^{(n)} \rightarrow \alpha r^{A} t^{B\{n\}} H_{A B}, \\
& H_{2}^{(n)} \rightarrow \alpha r^{A} r^{B\{n\}} H_{A B}, \\
& h_{0}^{(n)} \rightarrow \alpha f t^{A\{n\}} H_{A}, \\
& h_{1}^{(n)} \rightarrow \alpha f^{-1} r^{A\{n\}} H_{A},
\end{aligned}
$$




$$
\begin{gathered}
K^{(n)} \rightarrow \alpha\left(3^{\{n\}} G+{ }^{\{n\}} K\right), \\
G^{(n)} \rightarrow \alpha^{\{n\}} G .
\end{gathered}
$$

In our notation, the $n$ th-order Zerilli variable, in terms of the GS invariants, is given by

$$
{ }^{\{n\}} \Pi_{\mathrm{Z}} \equiv \frac{f r^{2}}{3 M+\lambda}\left(f r^{B\{n\}} \mathcal{K}_{A B}-r^{\{n\}} \mathcal{K}_{\mid A}\right) r^{A}+r^{\{n\}} \mathcal{K},
$$

with $\lambda \equiv \frac{1}{2}(l-1)(l+2)$. In particular, at second order we could add to this function any quadratic combination of first-order modes, which would simply change the source term of the Zerilli equation. Nevertheless, this is the simplest Zerilli variable one can define. Using the sources (67), (68), and (71)-(76) we have computed the second-order GS invariants, compared our Zerilli function (B13) with that found by Garat and Price, and verified their formula (41) of Ref. [8].
[1] D. Brizuela, J. M. Martín-García, and G. A. Mena Marugán, Phys. Rev. D 74, 044039 (2006).

[2] U.H. Gerlach and U. K. Sengupta, Phys. Rev. D 19, 2268 (1979).

[3] T. Regge and J. A. Wheeler, Phys. Rev. 108, 1063 (1957).

[4] R. Sachs, Relativity, Groups and Topology (Gordon and Breach, New York, 1964).

[5] V. Moncrief, Ann. Phys. (N.Y.) 88, 323 (1974).

[6] J. Stewart and M. Walker, Proc. R. Soc. A 341, 49 (1974).

[7] M. Bruni, S. Matarrese, S. Mollerach, and S. Sonego, Classical Quantum Gravity 14, 2585 (1997).

[8] A. Garat and R. H. Price, Phys. Rev. D 61, 044006 (2000).

[9] S. Sonego and M. Bruni, Commun. Math. Phys. 193, 209 (1998).

[10] C.T. Cunningham, R.H. Price, and V. Moncrief, Astrophys. J. 236, 674 (1980).

[11] J. M. Stewart, Classical Quantum Gravity 7, 1169 (1990).

[12] M. Karlovini, Classical Quantum Gravity 19, 2125 (2002).

[13] B. Nolan, Phys. Rev. D 70, 044004 (2004).

[14] V. Moncrief, Phys. Rev. D 9, 2707 (1974).

[15] O. Sarbach and M. Tiglio, Phys. Rev. D 64, 084016 (2001).
[16] M. Campanelli and C. O. Lousto, Phys. Rev. D 59, 124022 (1999).

[17] H. Goldstein, Classical Mechanics (Addison-Wesley, Reading, MA, 1980), 2nd ed.

[18] C. Rovelli, Phys. Rev. D 43, 442 (1991).

[19] J. M. Bardeen, Phys. Rev. D 22, 1882 (1980).

[20] R. Gleiser, C. Nicasio, R. Price, and J. Pullin, Phys. Rep. 325, 41 (2000).

[21] K. A. Malik and D. Wands, Classical Quantum Gravity 21, L65 (2004).

[22] K. Nakamura, Prog. Theor. Phys. 110, 723 (2003).

[23] F. J. Zerilli, J. Math. Phys. (N.Y.) 11, 2203 (1970).

[24] A. Campolattaro and K. S. Thorne, Astrophys. J. 159, 847 (1970).

[25] H. Kodama, A. Ishibashi, and O. Seto, Phys. Rev. D 62, 064022 (2000).

[26] xTensor, a free package for tensor manipulations in Mathematica, José M. Martín-García. It can be downloaded under the GNU General Public License from http://metric.iem.csic.es/Martin-Garcia/xAct.

[27] C. Gundlach and J. M. Martín-García, Phys. Rev. D 61, 084024 (2000). 